Article

\title{
Strong Convergence of Extragradient-Type Method to Solve Pseudomonotone Variational Inequalities Problems
}

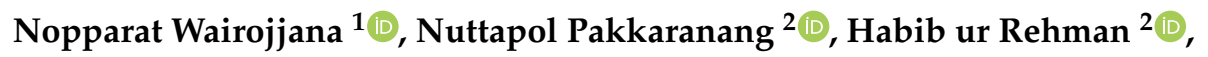 \\ Nattawut Pholasa ${ }^{3, *} *$ and Tiwabhorn Khanpanuk ${ }^{4, *}$ \\ 1 Applied Mathematics Program, Faculty of Science and Technology, Valaya Alongkorn Rajabhat University \\ under the Royal Patronage (VRU), 1 Moo 20 Phaholyothin Road, Klong Neung, Klong Luang, \\ Pathumthani 13180, Thailand; nopparat@vru.ac.th \\ 2 Department of Mathematics, Faculty of Science, King Mongkut's University of Technology \\ Thonburi (KMUTT), Bangkok 10140, Thailand; nuttapol.pak@mail.kmutt.ac.th (N.P.); \\ habib.rehman@mail.kmutt.ac.th (H.u.R.) \\ 3 School of Science, University of Phayao, Phayao 56000, Thailand \\ 4 Department of Mathematics, Faculty of Science and Technology, Phetchabun Rajabhat University, \\ Phetchabun 67000, Thailand \\ * Correspondence: nattawut_math@hotmail.com (N.P.); mathiproof@gmail.com (T.K.)
}

Received: 23 August 2020; Accepted: 30 September 2020; Published: 13 October 2020

\begin{abstract}
A number of applications from mathematical programmings, such as minimax problems, penalization methods and fixed-point problems can be formulated as a variational inequality model. Most of the techniques used to solve such problems involve iterative algorithms, and that is why, in this paper, we introduce a new extragradient-like method to solve the problems of variational inequalities in real Hilbert space involving pseudomonotone operators. The method has a clear advantage because of a variable stepsize formula that is revised on each iteration based on the previous iterations. The key advantage of the method is that it works without the prior knowledge of the Lipschitz constant. Strong convergence of the method is proved under mild conditions. Several numerical experiments are reported to show the numerical behaviour of the method.
\end{abstract}

Keywords: pseudomonotone mapping; subgradient extragradient method; strong convergence; Hilbert spaces; variational inequality problems

\section{Introduction}

In this article, we consider the classic variational inequalities problems (VIPs) [1,2] for an operator $\mathcal{F}: \mathcal{E} \rightarrow \mathcal{E}$ is formulated in the following way:

$$
\text { Find } u^{*} \in \mathcal{K} \text { such that }\left\langle\mathcal{F}\left(u^{*}\right), y-u^{*}\right\rangle \geq 0, \forall y \in \mathcal{K} \text {, }
$$

where $\mathcal{K}$ is a nonempty, convex and closed subset of a real Hilbert space $\mathcal{E}$. The inner product and induced norm on $\mathcal{E}$ are denoted by $\langle.,$.$\rangle and \|$.$\| , respectively. Moreover, the set of real and natural$ numbers are denoted by $\mathcal{R}$ and $\mathcal{N}$, respectively. It is important to note that solving the problem (1) is equivalent to solving the following problem:

$$
\text { Find an element } u^{*} \in \mathcal{K} \text { such that } u^{*}=P_{\mathcal{K}}\left[u^{*}-\zeta \mathcal{F}\left(u^{*}\right)\right] \text {. }
$$

We assume that the following requirements have been fulfilled: 
(B1) The solution set of the problem (1), represented by SVIP is nonempty.

(B2) A mapping $\mathcal{F}: \mathcal{E} \rightarrow \mathcal{E}$ is called to be pseudomonotone, i.e.,

$$
\left\langle\mathcal{F}\left(y_{1}\right), y_{2}-y_{1}\right\rangle \geq 0 \Longrightarrow\left\langle\mathcal{F}\left(y_{2}\right), y_{1}-y_{2}\right\rangle \leq 0, \forall y_{1}, y_{2} \in \mathcal{K}
$$

(B3) A mapping $\mathcal{F}: \mathcal{E} \rightarrow \mathcal{E}$ is said to be Lipschitz continuous, i.e., there exists $L>0$ such that

$$
\left\|\mathcal{F}\left(y_{1}\right)-\mathcal{F}\left(y_{2}\right)\right\| \leq L\left\|y_{1}-y_{2}\right\|, \forall y_{1}, y_{2} \in \mathcal{K}
$$

(B4) A mapping $\mathcal{F}: \mathcal{E} \rightarrow \mathcal{E}$ is called to be sequentially weakly continuous, i.e., $\left\{\mathcal{F}\left(u_{n}\right)\right\}$ converges weakly to $\mathcal{F}(u)$, where $\left\{u_{n}\right\}$ weakly converges to $u$.

The concept of variational inequalities has been used as a powerful tool to study different subjects, i.e., physics, engineering, economics and optimization theory. The problem (1) was firstly introduced by Stampacchia [1] in 1964 and also provided that this problem (1) is a crucial problem in nonlinear analysis. This is an efficient mathematical technique that integrates several key elements of applied mathematics, i.e., the problems of network equilibrium, the necessary optimality conditions, the complementarity problems and the systems of non-linear equations (for more details [3-9]). On the other hand, the projection methods are important to find the numerical solution of variational inequalities. Many authors have proposed and studied different projection methods to solve the problem of variational inequalities (see for more details [10-20]) and others in [21-32]. In particular, Karpelevich [10] and Antipin [33] introduced the following extragradient method:

$$
\left\{\begin{array}{l}
u_{n} \in \mathcal{K} \\
v_{n}=P_{\mathcal{K}}\left[u_{n}-\zeta \mathcal{F}\left(u_{n}\right)\right] \\
u_{n+1}=P_{\mathcal{K}}\left[u_{n}-\zeta \mathcal{F}\left(v_{n}\right)\right]
\end{array}\right.
$$

Recently, the subgradient extragradient algorithm was established by Censor et al. [12] for solving problem (1) in real Hilbert space. Their method has the form

$$
\left\{\begin{array}{l}
u_{n} \in \mathcal{K} \\
v_{n}=P_{\mathcal{K}}\left[u_{n}-\zeta \mathcal{F}\left(u_{n}\right)\right] \\
u_{n+1}=P_{\mathcal{E}_{n}}\left[u_{n}-\zeta \mathcal{F}\left(v_{n}\right)\right]
\end{array}\right.
$$

where $\mathcal{E}_{n}=\left\{z \in \mathcal{E}:\left\langle u_{n}-\zeta \mathcal{F}\left(u_{n}\right)-v_{n}, z-v_{n}\right\rangle \leq 0\right\}$. Migorski et al. [34] proposed a viscosity-type subgradient extragradient method to solve monotone variational inequalities problems. The main contribution is the presence of a viscosity scheme in the algorithm that was used to improve the convergence rate of the iterative sequence and provide strong convergence theorem. The iterative sequence $\left\{u_{n}\right\}$ was generated in the following way: (i) Let $u_{0} \in \mathcal{K}, \mu \in(0,1), \zeta_{0}>0$ and a sequence $\gamma_{n} \subset(0,1)$ with $\gamma_{n} \rightarrow 0$ and $\sum_{n}^{\infty} \gamma_{n}=+\infty$. (ii) Compute

$$
\left\{\begin{array}{l}
v_{n}=P_{\mathcal{K}}\left[u_{n}-\zeta_{n} \mathcal{F}\left(u_{n}\right)\right] \\
w_{n}=P_{\mathcal{E}_{n}}\left[u_{n}-\zeta_{n} \mathcal{F}\left(v_{n}\right)\right] \\
u_{n+1}=\gamma_{n} f\left(u_{n}\right)+\left(1-\gamma_{n}\right) w_{n}
\end{array}\right.
$$

where

$$
\mathcal{E}_{n}=\left\{z \in \mathcal{E}:\left\langle u_{n}-\zeta_{n} \mathcal{F}\left(u_{n}\right)-v_{n}, z-v_{n}\right\rangle \leq 0\right\} .
$$

(iii) Revised the stepsize in the following way:

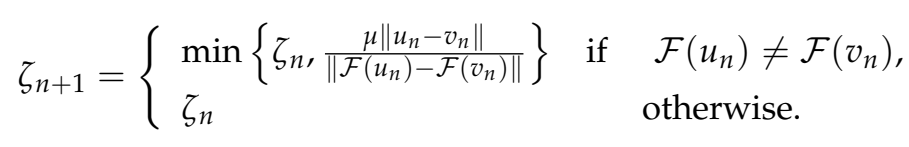


In this paper, inspired by the iterative methods in $[12,16,35,36]$, a modified subgradient extragradient algorithm is proposed for solving variational inequalities problems involving pseudomonotone mapping in real Hilbert space. It is important to note that our proposed scheme is effective. In particular, by comparing the results of Migorski et al. [34], our algorithm can solve pseudomonotone variational inequalities. Similar to the results of Migorski et al. [34] the proof of strong convergence of the proposed algorithm is proved without knowing the Lipschitz constant of the operator $\mathcal{F}$. The proposed algorithm could be seen as a modification of the methods that are appeared in $[10,12,34-36]$. Under mild conditions, a strong convergence theorem is proved. Numerical experiments have been shown that the new approach tends to be more successful than the existing one [34].

The rest of this article has been arranged as follows: Section 2 contains some definitions and basic results that have been used throughout the paper. Section 3 contains our main algorithm and a strong convergence theorem. Section 4 presents the numerical results showing the algorithmic efficacy of the proposed method.

\section{Preliminaries}

This section contains useful lemmas and basic identities that have been used throughout the article. The metric projection $P_{\mathcal{K}}\left(u_{1}\right)$ for $u_{1} \in \mathcal{E}$ onto a closed and convex subset $\mathcal{K}$ of $\mathcal{E}$ is defined by

$$
P_{\mathcal{K}}\left(u_{1}\right)=\arg \min \left\{\left\|u_{2}-u_{1}\right\|: u_{2} \in \mathcal{K}\right\}
$$

Lemma 1. [37,38] Assume $\mathcal{K}$ is a nonempty, convex and closed subset of a real Hilbert space $\mathcal{E}$ and $P_{\mathcal{K}}: \mathcal{E} \rightarrow \mathcal{K}$ is a metric projection from $\mathcal{E}$ onto $\mathcal{K}$.

(i) Let $u_{1} \in \mathcal{K}$ and $u_{2} \in \mathcal{E}$, we have

$$
\left\|u_{1}-P_{\mathcal{K}}\left(u_{2}\right)\right\|^{2}+\left\|P_{\mathcal{K}}\left(u_{2}\right)-u_{2}\right\|^{2} \leq\left\|u_{1}-u_{2}\right\|^{2} .
$$

(ii) $u_{3}=P_{\mathcal{K}}\left(u_{1}\right)$ if and only if

$$
\left\langle u_{1}-u_{3}, u_{2}-u_{3}\right\rangle \leq 0, \forall u_{2} \in \mathcal{K}
$$

(iii) For $u_{2} \in \mathcal{K}$ and $u_{1} \in \mathcal{E}$

$$
\left\|u_{1}-P_{\mathcal{K}}\left(u_{1}\right)\right\| \leq\left\|u_{1}-u_{2}\right\|
$$

Lemma 2. [37] Let $u, v \in \mathcal{E}$ and $\omega \in \mathcal{R}$.

(i) $\|\mathfrak{\omega} u+(1-\mathfrak{\omega}) v\|^{2}=\mathfrak{\omega}\|u\|^{2}+(1-\mathfrak{\omega})\|v\|^{2}-\mathfrak{\omega}(1-\mathfrak{\omega})\|u-v\|^{2}$.

(ii) $\|u+v\|^{2} \leq\|u\|^{2}+2\langle v, u+v\rangle$.

Lemma 3. [39] Assume that $\left\{\chi_{n}\right\}$ be a sequence of non-negative real numbers satisfying

$$
\chi_{n+1} \leq\left(1-\tau_{n}\right) \chi_{n}+\tau_{n} \delta_{n}, \forall n \in \mathcal{N},
$$

where $\left\{\tau_{n}\right\} \subset(0,1)$ and $\left\{\delta_{n}\right\} \subset \mathcal{R}$ satisfy the following conditions:

$$
\lim _{n \rightarrow \infty} \tau_{n}=0, \sum_{n=1}^{\infty} \tau_{n}=\infty, \text { and } \limsup _{n \rightarrow \infty} \delta_{n} \leq 0 .
$$

Then, $\lim _{n \rightarrow \infty} \chi_{n}=0$. 
Lemma 4. [40] Assume that $\left\{\chi_{n}\right\}$ is a sequence of real numbers such that there exists a subsequence $\left\{n_{i}\right\}$ of $\{n\}$ such that $\chi_{n_{i}}<\chi_{n_{i+1}}$ for all $i \in \mathcal{N}$. Then, there exists a non decreasing sequence $m_{k} \subset \mathcal{N}$ such that $m_{k} \rightarrow \infty$ as $k \rightarrow \infty$, and the following conditions are fulfilled by all (sufficiently large) numbers $k \in \mathcal{N}$ :

$$
\chi_{m_{k}} \leq \chi_{m_{k+1}} \text { and } \chi_{k} \leq \chi_{m_{k+1}} .
$$

In fact, $m_{k}=\max \left\{j \leq k: \chi_{j} \leq \chi_{j+1}\right\}$.

Lemma 5. [41] Assume that $\mathcal{F}: \mathcal{K} \rightarrow \mathcal{E}$ is a pseudomonotone and continuous mapping. Then, $u^{*}$ is a solution of the problem (1) if and only if $u^{*}$ is a solution of the following problem.

Find $x \in \mathcal{K}$ such that $\langle\mathcal{F}(y), y-x\rangle \geq 0, \forall y \in \mathcal{K}$.

\section{Main Results}

We provide a method consisting of two convex minimization problems through a viscosity scheme and an explicit stepsize formula which is being used to improve the convergence rate of the iterative sequence and to make the method independent of the Lipschitz constants. The detailed method is provided in Algorithm 1.

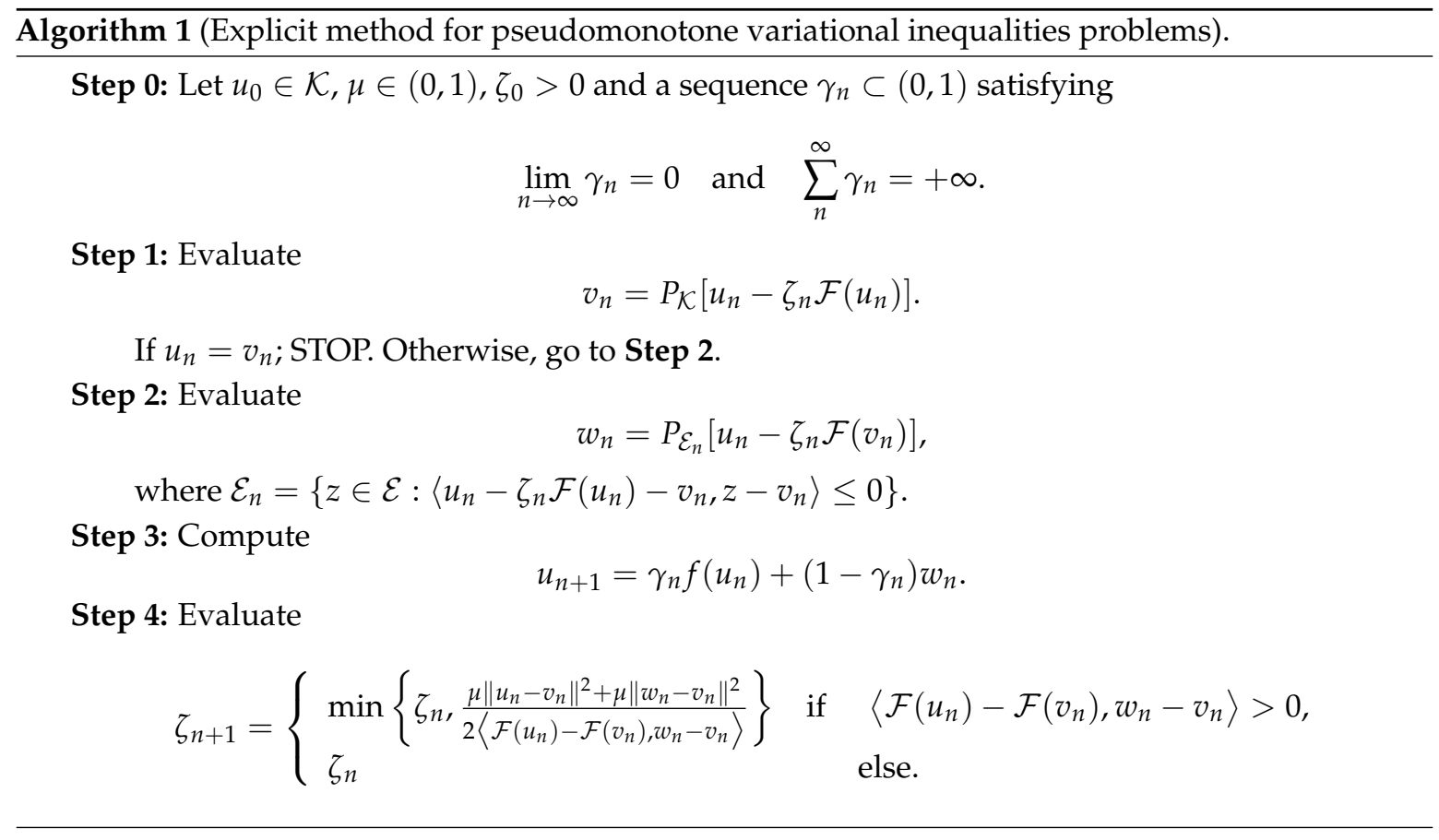

Lemma 6. The stepsize sequence $\left\{\zeta_{n}\right\}$ is monotonically decreasing with a lower bound $\min \left\{\frac{\mu}{L}, \zeta_{0}\right\}$ and converges to a fixed $\zeta>0$.

Proof. Let $\left\langle\mathcal{F}\left(u_{n}\right)-\mathcal{F}\left(v_{n}\right), w_{n}-v_{n}\right\rangle>0$, such that

$$
\begin{aligned}
\frac{\mu\left(\left\|u_{n}-v_{n}\right\|^{2}+\left\|w_{n}-v_{n}\right\|^{2}\right)}{2\left\langle\mathcal{F}\left(u_{n}\right)-\mathcal{F}\left(v_{n}\right), w_{n}-v_{n}\right\rangle} & \geq \frac{2 \mu\left\|u_{n}-v_{n}\right\|\left\|w_{n}-v_{n}\right\|}{2\left\|\mathcal{F}\left(u_{n}\right)-\mathcal{F}\left(v_{n}\right)\right\|\left\|w_{n}-v_{n}\right\|} \\
& \geq \frac{2 \mu\left\|u_{n}-v_{n}\right\|\left\|w_{n}-v_{n}\right\|}{2\left\|u_{n}-v_{n}\right\|\left\|w_{n}-v_{n}\right\|} \\
& \geq \frac{\mu}{L} .
\end{aligned}
$$


Clearly, from above we can conclude that $\left\{\zeta_{n}\right\}$ has a lower bound $\min \left\{\frac{\mu}{L}, \zeta_{0}\right\}$. Moreover, there exists a real number $\zeta>0$, such that $\lim _{n \rightarrow \infty} \zeta_{n}=\zeta$.

Lemma 7. Assume that $\mathcal{F}: \mathcal{E} \rightarrow \mathcal{E}$ satisfies the conditions (B1)-(B4). For a given $u^{*} \in S V I P \neq \varnothing$, we have

$$
\left\|w_{n}-u^{*}\right\|^{2} \leq\left\|u_{n}-u^{*}\right\|^{2}-\left(1-\frac{\mu \zeta_{n}}{\zeta_{n+1}}\right)\left\|u_{n}-v_{n}\right\|^{2}-\left(1-\frac{\mu \zeta_{n}}{\zeta_{n+1}}\right)\left\|w_{n}-v_{n}\right\|^{2} .
$$

Proof. Consider that

$$
\begin{aligned}
\left\|w_{n}-u^{*}\right\|^{2} & =\left\|P_{\mathcal{E}_{n}}\left[u_{n}-\zeta_{n} \mathcal{F}\left(v_{n}\right)\right]-u^{*}\right\|^{2} \\
& =\left\|P_{\mathcal{E}_{n}}\left[u_{n}-\zeta_{n} \mathcal{F}\left(v_{n}\right)\right]+\left[u_{n}-\zeta_{n} \mathcal{F}\left(v_{n}\right)\right]-\left[u_{n}-\zeta_{n} \mathcal{F}\left(v_{n}\right)\right]-u^{*}\right\|^{2} \\
& =\left\|\left[u_{n}-\zeta_{n} \mathcal{F}\left(v_{n}\right)\right]-u^{*}\right\|^{2}+\left\|P_{\mathcal{E}_{n}}\left[u_{n}-\zeta_{n} \mathcal{F}\left(v_{n}\right)\right]-\left[u_{n}-\zeta_{n} \mathcal{F}\left(v_{n}\right)\right]\right\|^{2} \\
& +2\left\langle P_{\mathcal{E}_{n}}\left[u_{n}-\zeta_{n} \mathcal{F}\left(v_{n}\right)\right]-\left[u_{n}-\zeta_{n} \mathcal{F}\left(v_{n}\right)\right],\left[u_{n}-\zeta_{n} \mathcal{F}\left(v_{n}\right)\right]-u^{*}\right\rangle .
\end{aligned}
$$

Given that $u^{*} \in S V I P \subset \mathcal{K} \subset \mathcal{E}_{n}$, we get

$$
\begin{aligned}
\| & P_{\mathcal{E}_{n}}\left[u_{n}-\zeta_{n} \mathcal{F}\left(v_{n}\right)\right]-\left[u_{n}-\zeta_{n} \mathcal{F}\left(v_{n}\right)\right] \|^{2} \\
& +\left\langle P_{\mathcal{E}_{n}}\left[u_{n}-\zeta_{n} \mathcal{F}\left(v_{n}\right)\right]-\left[u_{n}-\zeta_{n} \mathcal{F}\left(v_{n}\right)\right],\left[u_{n}-\zeta_{n} \mathcal{F}\left(v_{n}\right)\right]-u^{*}\right\rangle \\
= & \left\langle\left[u_{n}-\zeta_{n} \mathcal{F}\left(v_{n}\right)\right]-P_{\mathcal{E}_{n}}\left[u_{n}-\zeta_{n} \mathcal{F}\left(v_{n}\right)\right], u^{*}-P_{\mathcal{E}_{n}}\left[u_{n}-\zeta_{n} \mathcal{F}\left(v_{n}\right)\right]\right\rangle \leq 0,
\end{aligned}
$$

which implies that

$$
\begin{aligned}
&\left\langle P_{\mathcal{E}_{n}}\left[u_{n}-\zeta_{n} \mathcal{F}\left(v_{n}\right)\right]-\left[u_{n}-\zeta_{n} \mathcal{F}\left(v_{n}\right)\right],\left[u_{n}-\zeta_{n} \mathcal{F}\left(v_{n}\right)\right]-u^{*}\right\rangle \\
& \leq-\left\|P_{\mathcal{E}_{n}}\left[u_{n}-\zeta_{n} \mathcal{F}\left(v_{n}\right)\right]-\left[u_{n}-\zeta_{n} \mathcal{F}\left(v_{n}\right)\right]\right\|^{2} .
\end{aligned}
$$

Using expressions (6) and (8), we obtain

$$
\begin{aligned}
\left\|w_{n}-u^{*}\right\|^{2} & \leq\left\|u_{n}-\zeta_{n} \mathcal{F}\left(v_{n}\right)-u^{*}\right\|^{2}-\left\|P_{\mathcal{E}_{n}}\left[u_{n}-\zeta_{n} \mathcal{F}\left(v_{n}\right)\right]-\left[u_{n}-\zeta_{n} \mathcal{F}\left(v_{n}\right)\right]\right\|^{2} \\
& \leq\left\|u_{n}-u^{*}\right\|^{2}-\left\|u_{n}-w_{n}\right\|^{2}+2 \zeta_{n}\left\langle\mathcal{F}\left(v_{n}\right), u^{*}-w_{n}\right\rangle .
\end{aligned}
$$

Since $u^{*}$ is the solution of problem (1), we have

$$
\left\langle\mathcal{F}\left(u^{*}\right), y-u^{*}\right\rangle \geq 0, \text { for all } y \in \mathcal{K} .
$$

Due to the pseudomonotonicity of $\mathcal{F}$ on $\mathcal{K}$, we get

$$
\left\langle\mathcal{F}(y), y-u^{*}\right\rangle \geq 0, \text { for all } y \in \mathcal{K} .
$$

By substituting $y=v_{n} \in \mathcal{K}$, we get

$$
\left\langle\mathcal{F}\left(v_{n}\right), v_{n}-u^{*}\right\rangle \geq 0
$$

Thus, we have

$$
\left\langle\mathcal{F}\left(v_{n}\right), u^{*}-w_{n}\right\rangle=\left\langle\mathcal{F}\left(v_{n}\right), u^{*}-v_{n}\right\rangle+\left\langle\mathcal{F}\left(v_{n}\right), v_{n}-w_{n}\right\rangle \leq\left\langle\mathcal{F}\left(v_{n}\right), v_{n}-w_{n}\right\rangle .
$$

Combining expressions (9) and (10), we obtain

$$
\begin{aligned}
\left\|w_{n}-u^{*}\right\|^{2} & \leq\left\|u_{n}-u^{*}\right\|^{2}-\left\|u_{n}-w_{n}\right\|^{2}+2 \zeta_{n}\left\langle\mathcal{F}\left(v_{n}\right), v_{n}-w_{n}\right\rangle \\
& \leq\left\|u_{n}-u^{*}\right\|^{2}-\left\|u_{n}-v_{n}+v_{n}-w_{n}\right\|^{2}+2 \zeta_{n}\left\langle\mathcal{F}\left(v_{n}\right), v_{n}-w_{n}\right\rangle \\
& \leq\left\|u_{n}-u^{*}\right\|^{2}-\left\|u_{n}-v_{n}\right\|^{2}-\left\|v_{n}-w_{n}\right\|^{2}+2\left\langle u_{n}-\zeta_{n} \mathcal{F}\left(v_{n}\right)-v_{n}, w_{n}-v_{n}\right\rangle .
\end{aligned}
$$


Note that $w_{n}=P_{\mathcal{E}_{n}}\left[u_{n}-\zeta_{n} \mathcal{F}\left(v_{n}\right)\right]$ and by the definition of $\zeta_{n+1}$, we have

$$
\begin{aligned}
& 2\left\langle u_{n}-\zeta_{n} \mathcal{F}\left(v_{n}\right)-v_{n}, w_{n}-v_{n}\right\rangle \\
& =2\left\langle u_{n}-\zeta_{n} \mathcal{F}\left(u_{n}\right)-v_{n}, w_{n}-v_{n}\right\rangle+2 \zeta_{n}\left\langle\mathcal{F}\left(u_{n}\right)-\mathcal{F}\left(v_{n}\right), w_{n}-v_{n}\right\rangle \\
& \leq \frac{2 \zeta_{n}}{\zeta_{n+1}} \zeta_{n+1}\left\langle\mathcal{F}\left(u_{n}\right)-\mathcal{F}\left(v_{n}\right), w_{n}-v_{n}\right\rangle \leq \frac{\zeta_{n}}{\zeta_{n+1}}\left[\mu\left\|u_{n}-v_{n}\right\|^{2}+\mu\left\|w_{n}-v_{n}\right\|^{2}\right] .
\end{aligned}
$$

Combining expressions (11) and (12), we obtain

$$
\begin{aligned}
& \left\|w_{n}-u^{*}\right\|^{2} \\
& \leq\left\|u_{n}-u^{*}\right\|^{2}-\left\|u_{n}-v_{n}\right\|^{2}-\left\|v_{n}-w_{n}\right\|^{2}+\frac{\zeta_{n}}{\zeta_{n+1}}\left[\mu\left\|u_{n}-v_{n}\right\|^{2}+\mu\left\|w_{n}-v_{n}\right\|^{2}\right] \\
& \leq\left\|u_{n}-u^{*}\right\|^{2}-\left(1-\frac{\mu \zeta_{n}}{\zeta_{n+1}}\right)\left\|u_{n}-v_{n}\right\|^{2}-\left(1-\frac{\mu \zeta_{n}}{\zeta_{n+1}}\right)\left\|w_{n}-v_{n}\right\|^{2} .
\end{aligned}
$$

Lemma 8. Suppose that conditions (B1)-(B4) hold. Let $\left\{u_{n}\right\}$ be a sequence generated by Algorithm 1. If there is a subsequence $\left\{u_{n_{k}}\right\}$ which is weakly convergent to $\hat{u} \in \mathcal{E}$ and $\lim _{n \rightarrow \infty}\left\|u_{n}-v_{n}\right\|=0$, then $\hat{u} \in S V I P$.

Proof. We have

$$
v_{n_{k}}=P_{\mathcal{K}}\left[u_{n_{k}}-\zeta_{n_{k}} \mathcal{F}\left(u_{n_{k}}\right)\right],
$$

which is equivalent to

$$
\left\langle u_{n_{k}}-\zeta_{n_{k}} \mathcal{F}\left(u_{n_{k}}\right)-v_{n_{k}}, y-v_{n_{k}}\right\rangle \leq 0, \forall y \in \mathcal{K} .
$$

From expression (15), we can write

$$
\left\langle u_{n_{k}}-v_{n_{k}}, y-v_{n_{k}}\right\rangle \leq \zeta_{n_{k}}\left\langle\mathcal{F}\left(u_{n_{k}}\right), y-v_{n_{k}}\right\rangle, \forall y \in \mathcal{K} .
$$

Therefore, we get

$$
\frac{1}{\zeta_{n_{k}}}\left\langle u_{n_{k}}-v_{n_{k}}, y-v_{n_{k}}\right\rangle+\left\langle\mathcal{F}\left(u_{n_{k}}\right), v_{n_{k}}-u_{n_{k}}\right\rangle \leq\left\langle\mathcal{F}\left(u_{n_{k}}\right), y-u_{n_{k}}\right\rangle, \forall y \in \mathcal{K} .
$$

Due to the boundedness of the sequence $\left\{u_{n_{k}}\right\}$ so does $\left\{\mathcal{F}\left(u_{n_{k}}\right)\right\}$. By using the facts $\lim _{n \rightarrow \infty} \| u_{n_{k}}-$ $v_{n_{k}} \|=0$, and $\lim _{k \rightarrow \infty} \zeta_{n_{k}}=\zeta>0$, limit as $k \rightarrow \infty$ in (17), we get

$$
\liminf _{k \rightarrow \infty}\left\langle\mathcal{F}\left(u_{n_{k}}\right), y-u_{n_{k}}\right\rangle \geq 0, \forall y \in \mathcal{K} .
$$

Moreover, we have

$$
\left\langle\mathcal{F}\left(v_{n_{k}}\right), y-v_{n_{k}}\right\rangle=\left\langle\mathcal{F}\left(v_{n_{k}}\right)-\mathcal{F}\left(u_{n_{k}}\right), y-u_{n_{k}}\right\rangle+\left\langle\mathcal{F}\left(u_{n_{k}}\right), y-u_{n_{k}}\right\rangle+\left\langle\mathcal{F}\left(v_{n_{k}}\right), u_{n_{k}}-v_{n_{k}}\right\rangle .
$$

Since $\lim _{n \rightarrow \infty}\left\|u_{n_{k}}-v_{n_{k}}\right\|=0$, and $\mathcal{F}$ is L-Lipschitz continuous on $\mathcal{E}$, we get

$$
\lim _{n \rightarrow \infty}\left\|\mathcal{F}\left(u_{n_{k}}\right)-\mathcal{F}\left(v_{n_{k}}\right)\right\|=0 .
$$

From (19) and (20), we obtain

$$
\liminf _{k \rightarrow \infty}\left\langle\mathcal{F}\left(v_{n_{k}}\right), y-v_{n_{k}}\right\rangle \geq 0, \forall y \in \mathcal{K}
$$


Next, we show that $u^{*} \in S V I P$. We choose a sequence $\left\{\epsilon_{k}\right\}$ of positive numbers decreasing and tending to 0 . For each $k$, we denote by $m_{k}$ the smallest positive integer such that

$$
\liminf _{k \rightarrow \infty}\left\langle\mathcal{F}\left(u_{n_{i}}\right), y-u_{n_{i}}\right\rangle+\epsilon_{k} \geq 0, \forall i \geq m_{k}
$$

Due to $\left\{\epsilon_{k}\right\}$ being decreasing, the sequence $\left\{m_{k}\right\}$ is increasing.

Case 1: If there is a subsequence $u_{n_{m_{k_{j}}}}$ of $u_{n_{m_{k}}}$ such that $\mathcal{F}\left(u_{n_{m_{k_{j}}}}\right)=0(\forall j)$. Letting $j \rightarrow \infty$, we obtain

$$
\left\langle\mathcal{F}\left(u^{*}\right), y-u^{*}\right\rangle=\lim _{j \rightarrow \infty}\left\langle\mathcal{F}\left(u_{n_{m_{k_{j}}}}\right), y-u^{*}\right\rangle=0 \text {. }
$$

Hence $u^{*} \in \mathcal{K}$, therefore we have $u^{*} \in S V I P$.

Case 2: If there exists $N_{0}$ such that for all $n_{m_{k}} \geq N_{0}, \mathcal{F}\left(u_{n_{m_{k}}}\right) \neq 0$. Suppose that

$$
\Theta_{n_{m_{k}}}=\frac{\mathcal{F}\left(u_{n_{m_{k}}}\right)}{\left\|\mathcal{F}\left(u_{n_{m_{k}}}\right)\right\|^{2}}, \forall n_{m_{k}} \geq N_{0}
$$

Due to the above definition, we obtain

$$
\left\langle\mathcal{F}\left(u_{n_{m_{k}}}\right), \mathcal{F}\left(\Theta_{n_{m_{k}}}\right)\right\rangle=1, \forall n_{m_{k}} \geq N_{0} .
$$

From (18) and (25), for all $n_{m_{k}} \geq N_{0}$, we have

$$
\left\langle\mathcal{F}\left(u_{n_{m_{k}}}\right), y+\epsilon_{k} \Theta_{n_{m_{k}}}-u_{n_{m_{k}}}\right\rangle \geq 0 .
$$

Due to pseudomonotonicity of $\mathcal{F}$ for $n_{m_{k}} \geq N_{0}$, we obtain

$$
\left\langle\mathcal{F}\left(y+\epsilon_{k} \Theta_{n_{m_{k}}}\right), y+\epsilon_{k} \Theta_{n_{m_{k}}}-u_{n_{m_{k}}}\right\rangle \geq 0 .
$$

For all $n_{m_{k}} \geq N_{0}$, we have

$$
\left\langle\mathcal{F}(y), y-u_{n_{m_{k}}}\right\rangle \geq\left\langle\mathcal{F}(y)-\mathcal{F}\left(y+\epsilon_{k} \Theta_{n_{m_{k}}}\right), y+\epsilon_{k} \Theta_{n_{m_{k}}}-u_{n_{m_{k}}}\right\rangle-\epsilon_{k}\left\langle\mathcal{F}(y), \Theta_{n_{m_{k}}}\right\rangle .
$$

Since $\left\{u_{n_{k}}\right\}$ converges weakly to $u^{*} \in \mathcal{K}$ and $\mathcal{F}$ is sequentially weakly continuous on $\mathcal{K}$, we have $\left\{\mathcal{F}\left(u_{n_{k}}\right)\right\}$ converges weakly to $\mathcal{F}\left(u^{*}\right)$. We can suppose that $\mathcal{F}\left(u^{*}\right) \neq 0$. Since the norm mapping is sequentially weakly lower semicontinuous, we have

$$
\left\|\mathcal{F}\left(u^{*}\right)\right\| \leq \liminf _{k \rightarrow \infty}\left\|\mathcal{F}\left(u_{n_{k}}\right)\right\|
$$

Since $\left\{u_{n_{m_{k}}}\right\} \subset\left\{u_{n_{k}}\right\}$ and $\lim _{k \rightarrow \infty} \epsilon_{k}=0$, we have

$$
0 \leq \lim _{k \rightarrow \infty}\left\|\epsilon_{k} \Theta_{n_{m_{k}}}\right\|=\lim _{k \rightarrow \infty} \frac{\epsilon_{k}}{\left\|\mathcal{F}\left(u_{n_{m_{k}}}\right)\right\|} \leq \frac{0}{\left\|\mathcal{F}\left(u^{*}\right)\right\|}=0 .
$$

Now, letting $k \rightarrow \infty$ in (28), we obtain

$$
\left\langle\mathcal{F}(y), y-u^{*} \geq 0, \forall y \in \mathcal{K} .\right.
$$

Applying the well-known Lemma 5, we can deduce that $u^{*} \in S V I P$.

Theorem 1. Assume that $\mathcal{F}: \mathcal{K} \rightarrow \mathcal{E}$ satisfies the conditions (B1)-(B4). Moreover, assume that $u^{*}$ belongs to the solution set SVIP. Then, the sequences $\left\{u_{n}\right\},\left\{v_{n}\right\}$ and $\left\{w_{n}\right\}$ generated by Algorithm 1 converge strongly to $u^{*}$. 
Proof. By using Lemma 7, we have

$$
\left\|w_{n}-u^{*}\right\|^{2} \leq\left\|u_{n}-u^{*}\right\|^{2}-\left(1-\frac{\mu \zeta_{n}}{\zeta_{n+1}}\right)\left\|u_{n}-v_{n}\right\|^{2}-\left(1-\frac{\mu \zeta_{n}}{\zeta_{n+1}}\right)\left\|w_{n}-v_{n}\right\|^{2} .
$$

Due to $\zeta_{n} \rightarrow \zeta$, there exists a fixed number $\epsilon \in(0,1-\mu)$ such that

$$
\lim _{n \rightarrow \infty}\left(1-\frac{\mu \zeta_{n}}{\zeta_{n+1}}\right)=1-\mu>\epsilon>0
$$

Then, there exists a finite number $N_{1} \in \mathcal{N}$ such that

$$
\left(1-\frac{\mu \zeta_{n}}{\zeta_{n+1}}\right)>\epsilon>0, \forall n \geq N_{1}
$$

Hence, we obtain

$$
\left\|w_{n}-u^{*}\right\|^{2} \leq\left\|u_{n}-u^{*}\right\|^{2}, \forall n \geq N_{1} .
$$

From the definition of the sequence $\left\{u_{n+1}\right\}$ and the fact that $f$ is a contraction with constant $\rho \in[0,1)$ and $n \geq N_{1}$, we obtain

$$
\begin{aligned}
\left\|u_{n+1}-u^{*}\right\| & =\left\|\gamma_{n} f\left(u_{n}\right)+\left(1-\gamma_{n}\right) w_{n}-u^{*}\right\| \\
& =\left\|\gamma_{n}\left[f\left(u_{n}\right)-u^{*}\right]+\left(1-\gamma_{n}\right)\left[w_{n}-u^{*}\right]\right\| \\
& =\left\|\gamma_{n}\left[f\left(u_{n}\right)+f\left(u^{*}\right)-f\left(u^{*}\right)-u^{*}\right]+\left(1-\gamma_{n}\right)\left[w_{n}-u^{*}\right]\right\| \\
& \leq \gamma_{n}\left\|f\left(u_{n}\right)-f\left(u^{*}\right)\right\|+\gamma_{n}\left\|f\left(u^{*}\right)-u^{*}\right\|+\left(1-\gamma_{n}\right)\left\|w_{n}-u^{*}\right\| \\
& \leq \gamma_{n} \rho\left\|u_{n}-u^{*}\right\|+\gamma_{n}\left\|f\left(u^{*}\right)-u^{*}\right\|+\left(1-\gamma_{n}\right)\left\|w_{n}-u^{*}\right\| .
\end{aligned}
$$

From expressions (34) and (36) and $\gamma_{n} \subset(0,1)$, we obtain

$$
\begin{aligned}
\left\|u_{n+1}-u^{*}\right\| & \leq \gamma_{n} \rho\left\|u_{n}-u^{*}\right\|+\gamma_{n}\left\|f\left(u^{*}\right)-u^{*}\right\|+\left(1-\gamma_{n}\right)\left\|u_{n}-u^{*}\right\| \\
& =\left[1-\gamma_{n}+\rho \gamma_{n}\right]\left\|u_{n}-u^{*}\right\|+\gamma_{n}(1-\rho) \frac{\left\|f\left(u^{*}\right)-u^{*}\right\|}{(1-\rho)} \\
& \leq \max \left\{\left\|u_{n}-u^{*}\right\|, \frac{\left\|f\left(u^{*}\right)-u^{*}\right\|}{(1-\rho)}\right\} \\
& \leq \max \left\{\left\|u_{N_{1}}-u^{*}\right\|, \frac{\left\|f\left(u^{*}\right)-u^{*}\right\|}{(1-\rho)}\right\} .
\end{aligned}
$$

Hence, we conclude that the sequence $\left\{u_{n}\right\}$ is bounded. Next, the reflexivity of $\mathcal{E}$ and the boundedness of the sequence $\left\{u_{n}\right\}$ guarantee that there exists a subsequence $\left\{u_{n_{k}}\right\}$ such that $\left\{u_{n_{k}}\right\} \rightarrow$ $u^{*} \in \mathcal{E}$ as $k \rightarrow \infty$. Now, we prove the strong convergence of the sequence iterative sequence $\left\{u_{n}\right\}$ generated by Algorithm 1 . Due to the continuity and pseudomonotonicity of the operator $\mathcal{F}$ imply that the solution set SVIP is a closed and convex set (for more details see [42,43]). Since the mapping $f$ is a contraction, $P_{S V I P} \circ f$ is a contraction. The Banach contraction theorem guarantee the existence of a fixed point of $u^{*} \in S V I P$ such that

$$
u^{*}=P_{S V I P}\left(f\left(u^{*}\right)\right)
$$

By using Lemma 1 (ii), we have

$$
\left\langle f\left(u^{*}\right)-u^{*}, y-u^{*}\right\rangle \leq 0, \forall y \in S V I P .
$$


From given $u_{n+1}=\gamma_{n} f\left(u_{n}\right)+\left(1-\gamma_{n}\right) w_{n}$, and using Lemma 2 (i) and Lemma 7, we have

$$
\begin{aligned}
&\left\|u_{n+1}-u^{*}\right\|^{2} \\
&=\left\|\gamma_{n} f\left(u_{n}\right)+\left(1-\gamma_{n}\right) w_{n}-u^{*}\right\|^{2} \\
&=\left\|\gamma_{n}\left[f\left(u_{n}\right)-u^{*}\right]+\left(1-\gamma_{n}\right)\left[w_{n}-u^{*}\right]\right\|^{2} \\
&=\gamma_{n}\left\|f\left(u_{n}\right)-u^{*}\right\|^{2}+\left(1-\gamma_{n}\right)\left\|w_{n}-u^{*}\right\|^{2}-\gamma_{n}\left(1-\gamma_{n}\right)\left\|f\left(u_{n}\right)-w_{n}\right\|^{2} \\
& \leq \gamma_{n}\left\|f\left(u_{n}\right)-u^{*}\right\|^{2}+\left(1-\gamma_{n}\right)\left[\left\|u_{n}-u^{*}\right\|^{2}-\left(1-\frac{\mu \zeta_{n}}{\zeta_{n+1}}\right)\left\|u_{n}-v_{n}\right\|^{2}\right. \\
&\left.-\left(1-\frac{\mu \zeta_{n}}{\zeta_{n+1}}\right)\left\|w_{n}-v_{n}\right\|^{2}\right]-\gamma_{n}\left(1-\gamma_{n}\right)\left\|f\left(u_{n}\right)-w_{n}\right\|^{2} \\
& \leq \gamma_{n}\left\|f\left(u_{n}\right)-u^{*}\right\|^{2}+\left\|u_{n}-u^{*}\right\|^{2}-\left(1-\gamma_{n}\right)\left(1-\frac{\mu \zeta_{n}}{\zeta_{n+1}}\right)\left[\left\|w_{n}-v_{n}\right\|^{2}+\left\|u_{n}-v_{n}\right\|^{2}\right] .
\end{aligned}
$$

The rest of the proof shall be divided into the following two parts:

Case 1: Assume that there exists a fixed number $N_{2} \in \mathcal{N}\left(N_{2} \geq N_{1}\right)$ such that

$$
\left\|u_{n+1}-u^{*}\right\| \leq\left\|u_{n}-u^{*}\right\|, \forall n \geq N_{2}
$$

Thus, $\lim _{n \rightarrow \infty}\left\|u_{n}-u^{*}\right\|$ exists and let $\lim _{n \rightarrow \infty}\left\|u_{n}-u^{*}\right\|=l$. From expression (38), we have

$$
\begin{aligned}
& \left(1-\gamma_{n}\right)\left(1-\frac{\mu \zeta_{n}}{\zeta_{n+1}}\right)\left[\left\|w_{n}-v_{n}\right\|^{2}+\left\|u_{n}-v_{n}\right\|^{2}\right] \\
& \leq \gamma_{n}\left\|f\left(u_{n}\right)-u^{*}\right\|^{2}+\left\|u_{n}-u^{*}\right\|^{2}-\left\|u_{n+1}-u^{*}\right\|^{2} .
\end{aligned}
$$

Due to the existence of $\lim _{n \rightarrow \infty}\left\|u_{n}-u^{*}\right\|=l$, and $\gamma_{n} \rightarrow 0$, we deduce that

$$
\lim _{n \rightarrow \infty}\left\|u_{n}-v_{n}\right\|=\lim _{n \rightarrow \infty}\left\|w_{n}-v_{n}\right\|=0 .
$$

From expression (41), we have

$$
\lim _{n \rightarrow \infty}\left\|u_{n}-w_{n}\right\| \leq \lim _{n \rightarrow \infty}\left\|u_{n}-v_{n}\right\|+\lim _{n \rightarrow \infty}\left\|v_{n}-w_{n}\right\|=0 .
$$

It follows that

$$
\begin{aligned}
\left\|u_{n+1}-u_{n}\right\| & =\left\|\gamma_{n} f\left(u_{n}\right)+\left(1-\gamma_{n}\right) w_{n}-u_{n}\right\| \\
& =\left\|\gamma_{n}\left[f\left(u_{n}\right)-u_{n}\right]+\left(1-\gamma_{n}\right)\left[w_{n}-u_{n}\right]\right\| \\
& \leq \gamma_{n}\left\|f\left(u_{n}\right)-u_{n}\right\|+\left(1-\gamma_{n}\right)\left\|w_{n}-u_{n}\right\| \longrightarrow 0 .
\end{aligned}
$$

Thus, the sequences $\left\{u_{n}\right\},\left\{v_{n}\right\}$ and $\left\{w_{n}\right\}$ are bounded. Thus, we can take a subsequence $\left\{u_{n_{k}}\right\}$ of $\left\{u_{n}\right\}$ such that $\left\{u_{n_{k}}\right\}$ weakly converges to some $\hat{u} \in \mathcal{E}$. Moreover, due to $\left\|u_{n}-v_{n}\right\| \rightarrow 0$ and using Lemma 8 , we have $\hat{u} \in S V I P$. By following expression (37), we consider that

$$
\begin{aligned}
& \limsup _{n \rightarrow \infty}\left\langle f\left(u^{*}\right)-u^{*}, u_{n}-u^{*}\right\rangle \\
& =\limsup _{k \rightarrow \infty}\left\langle f\left(u^{*}\right)-u^{*}, u_{n_{k}}-u^{*}\right\rangle=\left\langle f\left(u^{*}\right)-u^{*}, \hat{u}-u^{*}\right\rangle \leq 0 .
\end{aligned}
$$

We have $\lim _{n \rightarrow \infty}\left\|u_{n+1}-u_{n}\right\|=0$. It follows (44) that

$$
\begin{aligned}
& \limsup _{n \rightarrow \infty}\left\langle f\left(u^{*}\right)-u^{*}, u_{n+1}-u^{*}\right\rangle \\
& \leq \limsup _{k \rightarrow \infty}\left\langle f\left(u^{*}\right)-u^{*}, u_{n+1}-u_{n}\right\rangle+\limsup _{k \rightarrow \infty}\left\langle f\left(u^{*}\right)-u^{*}, u_{n}-u^{*}\right\rangle \leq 0 .
\end{aligned}
$$


From Lemma 2 (ii) and Lemma 7 for all $n \geq N_{2}$, we get

$$
\begin{aligned}
& \left\|u_{n+1}-u^{*}\right\|^{2} \\
& =\left\|\gamma_{n} f\left(u_{n}\right)+\left(1-\gamma_{n}\right) w_{n}-u^{*}\right\|^{2} \\
& =\left\|\gamma_{n}\left[f\left(u_{n}\right)-u^{*}\right]+\left(1-\gamma_{n}\right)\left[w_{n}-u^{*}\right]\right\|^{2} \\
& \leq\left(1-\gamma_{n}\right)^{2}\left\|w_{n}-u^{*}\right\|^{2}+2 \gamma_{n}\left\langle f\left(u_{n}\right)-u^{*},\left(1-\gamma_{n}\right)\left[w_{n}-u^{*}\right]+\gamma_{n}\left[f\left(u_{n}\right)-u^{*}\right]\right\rangle \\
& =\left(1-\gamma_{n}\right)^{2}\left\|w_{n}-u^{*}\right\|^{2}+2 \gamma_{n}\left\langle f\left(u_{n}\right)-f\left(u^{*}\right)+f\left(u^{*}\right)-u^{*}, u_{n+1}-u^{*}\right\rangle \\
& =\left(1-\gamma_{n}\right)^{2}\left\|w_{n}-u^{*}\right\|^{2}+2 \gamma_{n}\left\langle f\left(u_{n}\right)-f\left(u^{*}\right), u_{n+1}-u^{*}\right\rangle+2 \gamma_{n}\left\langle f\left(u^{*}\right)-u^{*}, u_{n+1}-u^{*}\right\rangle \\
& \leq\left(1-\gamma_{n}\right)^{2}\left\|w_{n}-u^{*}\right\|^{2}+2 \gamma_{n} \rho\left\|u_{n}-u^{*}\right\|\left\|u_{n+1}-u^{*}\right\|+2 \gamma_{n}\left\langle f\left(u^{*}\right)-u^{*}, u_{n+1}-u^{*}\right\rangle \\
& \leq\left(1+\gamma_{n}^{2}-2 \gamma_{n}\right)\left\|u_{n}-u^{*}\right\|^{2}+2 \gamma_{n} \rho\left\|u_{n}-u^{*}\right\|^{2}+2 \gamma_{n}\left\langle f\left(u^{*}\right)-u^{*}, u_{n+1}-u^{*}\right\rangle \\
& =\left(1-2 \gamma_{n}\right)\left\|u_{n}-u^{*}\right\|^{2}+\gamma_{n}^{2}\left\|u_{n}-u^{*}\right\|^{2}+2 \gamma_{n} \rho\left\|u_{n}-u^{*}\right\|^{2}+2 \gamma_{n}\left\langle f\left(u^{*}\right)-u^{*}, u_{n+1}-u^{*}\right\rangle \\
& =\left[1-2 \gamma_{n}(1-\rho)\right]\left\|u_{n}-u^{*}\right\|^{2}+2 \gamma_{n}(1-\rho)\left[\frac{\gamma_{n}\left\|u_{n}-u^{*}\right\|^{2}}{2(1-\rho)}+\frac{\left\langle f\left(u^{*}\right)-u^{*}, u_{n+1}-u^{*}\right\rangle}{1-\rho}\right] .
\end{aligned}
$$

It follows from expressions (45) and (46), we obtain

$$
\limsup _{n \rightarrow \infty}\left[\frac{\gamma_{n}\left\|u_{n}-u^{*}\right\|^{2}}{2(1-\rho)}+\frac{\left\langle f\left(u^{*}\right)-u^{*}, u_{n+1}-u^{*}\right\rangle}{1-\rho}\right] \leq 0 .
$$

Choose $n \geq N_{3} \in \mathcal{N}\left(N_{3} \geq N_{2}\right)$ large enough such that $2 \gamma_{n}(1-\rho)<1$. Now, using expressions (46) and (47) and applying Lemma 3, we conclude that $\left\|u_{n}-u^{*}\right\| \rightarrow 0$, as $n \rightarrow \infty$. Case 2: Suppose that there exists a subsequence $\left\{n_{i}\right\}$ of $\{n\}$ such that

$$
\left\|u_{n_{i}}-u^{*}\right\| \leq\left\|u_{n_{i+1}}-u^{*}\right\|, \forall i \in \mathcal{N} .
$$

Thus, by Lemma 4 , there exits a sequence $\left\{m_{k}\right\} \subset \mathcal{N}$ and $\left\{m_{k}\right\} \rightarrow \infty$, such that

$$
\left\|u_{m_{k}}-u^{*}\right\| \leq\left\|u_{m_{k+1}}-u^{*}\right\| \quad \text { and } \quad\left\|u_{k}-u^{*}\right\| \leq\left\|u_{m_{k+1}}-u^{*}\right\|, \forall k \in \mathcal{N} .
$$

Similar to Case 1, using (38), we have

$$
\begin{aligned}
& \left(1-\gamma_{m_{k}}\right)\left(1-\frac{\mu \zeta_{m_{k}}}{\zeta_{m_{k}+1}}\right)\left[\left\|w_{m_{k}}-v_{m_{k}}\right\|^{2}+\left\|u_{m_{k}}-v_{m_{k}}\right\|^{2}\right] \\
& \leq \gamma_{m_{k}}\left\|f\left(u_{m_{k}}\right)-u^{*}\right\|^{2}+\left\|u_{m_{k}}-u^{*}\right\|^{2}-\left\|u_{m_{k}+1}-u^{*}\right\|^{2} .
\end{aligned}
$$

Due to $\gamma_{m_{k}} \rightarrow 0$ and $\left(1-\frac{\mu \zeta_{m_{k}}}{\zeta_{m_{k}+1}}\right) \rightarrow 1-\mu$, we can deduce the following:

$$
\lim _{n \rightarrow \infty}\left\|u_{m_{k}}-v_{m_{k}}\right\|=\lim _{k \rightarrow \infty}\left\|w_{m_{k}}-v_{m_{k}}\right\|=0 .
$$

From expression (50), we have

$$
\lim _{k \rightarrow \infty}\left\|u_{m_{k}}-w_{m_{k}}\right\| \leq \lim _{k \rightarrow \infty}\left\|u_{m_{k}}-v_{m_{k}}\right\|+\lim _{k \rightarrow \infty}\left\|v_{m_{k}}-w_{m_{k}}\right\|=0 .
$$


Hence, we obtain

$$
\begin{aligned}
\left\|u_{m_{k}+1}-u_{m_{k}}\right\| & =\left\|\gamma_{m_{k}} f\left(u_{m_{k}}\right)+\left(1-\gamma_{m_{k}}\right) w_{m_{k}}-u_{m_{k}}\right\| \\
& =\left\|\gamma_{m_{k}}\left[f\left(u_{m_{k}}\right)-u_{m_{k}}\right]+\left(1-\gamma_{m_{k}}\right)\left[w_{m_{k}}-u_{m_{k}}\right]\right\| \\
& \leq \gamma_{m_{k}}\left\|f\left(u_{m_{k}}\right)-u_{m_{k}}\right\|+\left(1-\gamma_{m_{k}}\right)\left\|w_{m_{k}}-u_{m_{k}}\right\| \longrightarrow 0
\end{aligned}
$$

We have to use the same justification as in the Case 1, such that

$$
\limsup _{k \rightarrow \infty}\left\langle f\left(u^{*}\right)-u^{*}, u_{m_{k}+1}-u^{*}\right\rangle \leq 0 .
$$

Using (46) and (48), we have

$$
\begin{aligned}
\left\|u_{m_{k}+1}-u^{*}\right\|^{2} \leq & {\left[1-2 \gamma_{m_{k}}(1-\rho)\right]\left\|u_{m_{k}}-u^{*}\right\|^{2} } \\
& +2 \gamma_{m_{k}}(1-\rho)\left[\frac{\gamma_{m_{k}}\left\|u_{m_{k}}-u^{*}\right\|^{2}}{2(1-\rho)}+\frac{\left\langle f\left(u^{*}\right)-u^{*}, u_{m_{k}+1}-u^{*}\right\rangle}{1-\rho}\right] \\
\leq & {\left[1-2 \gamma_{m_{k}}(1-\rho)\right]\left\|u_{m_{k}+1}-u^{*}\right\|^{2} } \\
& +2 \gamma_{m_{k}}(1-\rho)\left[\frac{\gamma_{m_{k}}\left\|u_{m_{k}}-u^{*}\right\|^{2}}{2(1-\rho)}+\frac{\left\langle f\left(u^{*}\right)-u^{*}, u_{m_{k}+1}-u^{*}\right\rangle}{1-\rho}\right] .
\end{aligned}
$$

It follows that

$$
\left\|u_{m_{k}+1}-u^{*}\right\|^{2} \leq \frac{\gamma_{m_{k}}\left\|u_{m_{k}}-u^{*}\right\|^{2}}{2(1-\rho)}+\frac{\left\langle f\left(u^{*}\right)-u^{*}, u_{m_{k}+1}-u^{*}\right\rangle}{1-\rho} .
$$

Since $\gamma_{m_{k}} \rightarrow 0$ and $\left\|u_{m_{k}}-u^{*}\right\|$ is a bounded sequence. Thus, expressions (53) and (55) implies that

$$
\left\|u_{m_{k}+1}-u^{*}\right\|^{2} \rightarrow 0 \text {, as } k \rightarrow \infty .
$$

From the inequality (48), we have

$$
\lim _{n \rightarrow \infty}\left\|u_{k}-u^{*}\right\|^{2} \leq \lim _{n \rightarrow \infty}\left\|u_{m_{k}+1}-u^{*}\right\|^{2} \leq 0 .
$$

Consequently, $u_{n} \rightarrow u^{*}$. This completes the proof of the theorem.

\section{Numerical Experiments}

Numerical investigations present in this section to demonstrate the efficiency of the introduced Algorithm 1 in four test problems, all of which are pseudomonotone. The MATLAB program has been performed on a PC (with Intel(R) Core(TM)i3-4010U CPU @ $1.70 \mathrm{GHz}$, RAM 4.00 GB) in MATLAB version 9.5 (R2018b). We use the built-in MATLAB Quadratic programming to solve the minimization problems.

Example 1. Consider the non-linear complementarity problem of Kojima--Shindo where the feasible set $\mathcal{K}$ which is defined by

$$
\mathcal{K}=\left\{u \in \mathcal{R}^{4}: 1 \leq u_{i} \leq 5, i=1,2,3,4\right\} .
$$


The mapping $\mathcal{F}: \mathcal{R}^{4} \rightarrow \mathcal{R}^{4}$ is defined by

$$
\mathcal{F}(u)=\left(\begin{array}{l}
u_{1}+u_{2}+u_{3}+u_{4}-4 u_{2} u_{3} u_{4} \\
u_{1}+u_{2}+u_{3}+u_{4}-4 u_{1} u_{3} u_{4} \\
u_{1}+u_{2}+u_{3}+u_{4}-4 u_{1} u_{2} u_{4} \\
u_{1}+u_{2}+u_{3}+u_{4}-4 u_{1} u_{2} u_{3}
\end{array}\right)
$$

It is easy to see that $\mathcal{F}$ is not monotone on the set $\mathcal{K}$. By using the Monte Carlo approach [44], it can be shown that $\mathcal{F}$ is pseudomonotone on $\mathcal{K}$, This problem has a unique solution $u^{*}=(5,5,5,5)^{T}$. Generate many pairs of points $u$ and $v$ uniformly in $\mathcal{K}$ satisfying $\mathcal{F}(u)^{T}(v-u) \geq 0$ and then check if $\mathcal{F}(v)^{T}(v-u) \geq 0$. In this experiment, we take different initial points and $D_{n}=\left\|u_{n}-v_{n}\right\|$. Moreover, control parameters $\zeta_{0}=0.33$, $\mu=0.25, \gamma_{n}=\frac{1}{100(n+2)}$ and $f(u)=\frac{u}{2}$ for Algorithm 1. Numerical investigation regarding the first example was shown in Table 1.

Table 1. Numerical behaviour of Algorithm 1 using different starting points for Example 1.

\begin{tabular}{lcccccccc}
\hline $\begin{array}{l}\text { TOL } \\
\boldsymbol{u}_{\mathbf{0}}\end{array}$ & $\begin{array}{c}\mathbf{1 0}^{-\mathbf{2}} \\
\text { Iter. }\end{array}$ & $\begin{array}{c}\mathbf{1 0}^{-\mathbf{3}} \\
\text { Iter. }\end{array}$ & $\begin{array}{r}\mathbf{1 0}^{-\mathbf{4}} \\
\text { Iter. }\end{array}$ & $\begin{array}{r}\mathbf{1 0}^{-\mathbf{5}} \\
\text { Iter. }\end{array}$ & $\begin{array}{c}\mathbf{1 0}^{-\mathbf{2}} \\
\text { Time }\end{array}$ & $\begin{array}{c}\mathbf{1 0}^{-3} \\
\text { Time }\end{array}$ & $\begin{array}{c}\mathbf{1 0}^{-\mathbf{4}} \\
\text { Time }\end{array}$ & $\begin{array}{l}\mathbf{1 0}^{-\mathbf{5}} \\
\text { Time }\end{array}$ \\
\hline$[-2,2,8,10]^{T}$ & 13 & 51 & 501 & 5001 & 0.079821 & 0.247776 & 3.251465 & 43.637834 \\
{$[-1,1,5,6]^{T}$} & 12 & 51 & 501 & 5001 & 0.083870 & 0.236924 & 2.684370 & 39.651178 \\
{$[-5,2,-1,2]^{T}$} & 9 & 51 & 501 & 5001 & 0.065422 & 0.235173 & 3.034747 & 43.630625 \\
{$[1,2,3,4]^{T}$} & 6 & 1004 & 1004 & 5001 & 0.040866 & 8.051234 & 6.686632 & 42.431705 \\
\hline
\end{tabular}

Example 2. Consider the quadratic fractional programming problem in the following form [44]:

$$
\left\{\begin{array}{l}
\min f(u)=\frac{u^{T} Q u+a^{T} u+a_{0}}{b^{T} u+b_{0}}, \\
\text { subject to } u \in \mathcal{K}=\left\{u \in \mathcal{R}^{4}: b^{T} u+b_{0}>0\right\},
\end{array}\right.
$$

where

$$
Q=\left(\begin{array}{cccc}
5 & -1 & 2 & 0 \\
-1 & 5 & -1 & 3 \\
2 & -1 & 3 & 0 \\
0 & 3 & 0 & 5
\end{array}\right), \quad a=\left(\begin{array}{c}
1 \\
-2 \\
-2 \\
1
\end{array}\right), \quad b=\left(\begin{array}{l}
2 \\
1 \\
1 \\
0
\end{array}\right), a_{0}=-2, \quad \text { and } \quad b_{0}=4
$$

It is easy to verify that $Q$ is symmetric and positive definite on $\mathcal{R}^{4}$ and consequently $f$ is pseudo-convex on $\mathcal{K}$. Therefore, $\nabla f$ is pseudomonotone. Using the quotient rule, we obtain

$$
\nabla f(u)=\frac{\left(b^{T} u+b_{0}\right)(2 Q u+a)-b\left(u^{T} Q+a^{T} u+a_{0}\right)}{\left(b^{T} u+b_{0}\right)^{2}} .
$$

In this point of view, we can set $\mathcal{F}=\nabla f$ in Theorem 1 . We minimize f over $\mathcal{K}=\left\{u \in \mathcal{R}^{4}: 1 \leq u_{i} \leq\right.$ $10, i=1,2,3,4\}$. This problem has a unique solution $u^{*}=(1,1,1,1)^{T} \in \mathcal{K}$. In this experiment, we take different initial points and $D_{n}=\left\|u_{n}-v_{n}\right\|$. Moreover, control parameters $\zeta_{0}=0.33, \mu=0.25, \gamma_{n}=\frac{1}{100(n+2)}$ and $f(u)=\frac{u}{2}$ for Algorithm 1. Numerical investigation regarding the second example is shown in Table 2.

Table 2. Numerical behaviour of Algorithm 1 using different starting points for Example 2.

\begin{tabular}{lcccccccc}
\hline TOL & $\begin{array}{c}\mathbf{1 0}^{-\mathbf{2}} \\
\text { Iter. }\end{array}$ & $\begin{array}{c}\mathbf{1 0}^{-\mathbf{3}} \\
\text { Iter. }\end{array}$ & $\begin{array}{c}\mathbf{1 0}^{-4} \\
\text { Iter. }\end{array}$ & $\begin{array}{c}\mathbf{1 0}^{-\mathbf{5}} \\
\text { Iter. }\end{array}$ & $\begin{array}{c}\mathbf{1 0}^{-\mathbf{2}} \\
\text { Time }\end{array}$ & $\begin{array}{c}\mathbf{1 0}^{-\mathbf{3}} \\
\text { Time }\end{array}$ & $\begin{array}{c}\mathbf{1 0}^{-\mathbf{4}} \\
\text { Time }\end{array}$ & $\begin{array}{c}\mathbf{1 0}^{-\mathbf{5}} \\
\text { Time }\end{array}$ \\
\hline$[10,10,10,10]^{T}$ & 43 & 46 & 99 & 989 & 0.289149 & 0.249285 & 0.475520 & 8.480530 \\
{$[10,20,30,40]^{T}$} & 41 & 46 & 99 & 989 & 0.211707 & 0.187559 & 0.445240 & 6.898924 \\
{$[20,-20,20,-20]^{T}$} & 29 & 32 & 99 & 989 & 0.138575 & 0.169190 & 0.394654 & 7.168460 \\
\hline
\end{tabular}


Example 3. The third example was taken from [45] where $\mathcal{F}: \mathcal{R}^{2} \rightarrow \mathcal{R}^{2}$ is defined by

$$
\mathcal{F}(u)=\left(\begin{array}{c}
0.5 u_{1} u_{2}-2 u_{2}-10^{7} \\
-4 u_{1}-0.1 u_{2}^{2}-10^{7}
\end{array}\right),
$$

on $\mathcal{K}=\left\{u \in \mathcal{R}^{2}:\left(u_{1}-2\right)^{2}+\left(u_{2}-2\right)^{2} \leq 1\right\}$. It can easily see that $\mathcal{F}$ is Lipschitz continuous with $L=5$ and $\mathcal{F}$ is not monotone on $\mathcal{K}$ but pseudomonotone. The above problem has a unique solution $u^{*}=(2.707,2.707)^{T}$. In this experiment, we take different initial points and $D_{n}=\left\|u_{n}-v_{n}\right\|$. Moreover, control parameters $\zeta_{0}=0.33$, $\mu=0.25, \gamma_{n}=\frac{1}{100(n+2)}$ and $f(u)=\frac{u}{3}$ for Algorithm 1. Numerical investigations regarding the third example is shown in Table 3.

Table 3. Numerical behaviour of Algorithm 1 using different starting points for Example 3.

\begin{tabular}{lcccccccc}
\hline $\begin{array}{l}\text { TOL } \\
\boldsymbol{u}_{\mathbf{0}}\end{array}$ & $\begin{array}{c}\mathbf{1 0}^{-\mathbf{2}} \\
\text { Iter. }\end{array}$ & $\begin{array}{c}\mathbf{1 0}^{-\mathbf{3}} \\
\text { Iter. }\end{array}$ & $\begin{array}{c}\mathbf{1 0}^{-\mathbf{4}} \\
\text { Iter. }\end{array}$ & $\begin{array}{l}\mathbf{1 0}^{-\mathbf{5}} \\
\text { Iter. }\end{array}$ & $\begin{array}{c}\mathbf{1 0}^{-\mathbf{2}} \\
\text { Time }\end{array}$ & $\begin{array}{c}\mathbf{1 0}^{-3} \\
\text { Time }\end{array}$ & $\begin{array}{c}\mathbf{1 0}^{-4} \\
\text { time }\end{array}$ & $\begin{array}{c}\mathbf{1 0}^{-\mathbf{5}} \\
\text { Time }\end{array}$ \\
\hline$[0,0]^{T}$ & 8 & 27 & 265 & 2566 & 0.606917 & 1.907212 & 14.120655 & 107.506926 \\
{$[10,10]^{T}$} & 7 & 27 & 265 & 2591 & 0.286659 & 1.057623 & 10.764532 & 116.258335 \\
{$[-5,-5]^{T}$} & 8 & 26 & 258 & 2596 & 0.388227 & 1.190191 & 11.424257 & 107.584978 \\
\hline
\end{tabular}

Example 4. The fourth example was taken from [45] where $\mathcal{F}: \mathcal{R}^{2} \rightarrow \mathcal{R}^{2}$ is defined by

$$
\mathcal{F}(u)=\left(\begin{array}{c}
\left(u_{1}^{2}+\left(u_{2}-1\right)^{2}\right)\left(1+u_{2}\right) \\
-u_{1}^{3}-u_{1}\left(u_{2}-1\right)^{2}
\end{array}\right)
$$

where $\mathcal{K}=\left\{u \in \mathcal{R}^{2}:-10 \leq u_{i} \leq 10, i=1,2\right\}$. It can easily see that $\mathcal{F}$ is Lipschitz continuous with $L=5$ and $\mathcal{F}$ is not monotone on $\mathcal{K}$ but pseudomonotone. In this experiment, we take different initial points and $D_{n}=\left\|u_{n}-v_{n}\right\|$. Moreover, control parameters $\zeta_{0}=0.33, \mu=0.25, \gamma_{n}=\frac{1}{100(n+2)}$ and $f(u)=\frac{u}{4}$ for Algorithm 1. Numerical investigations regarding the fourth example is shown in Table 4.

Table 4. Numerical behaviour of Algorithm 1 using different starting points for Example 4.

\begin{tabular}{lcclccccc}
\hline TOL & $\begin{array}{c}\mathbf{1 0}^{-\mathbf{2}} \\
\text { Iter. }\end{array}$ & $\begin{array}{c}\mathbf{1 0}^{-\mathbf{3}} \\
\text { Iter. }\end{array}$ & $\begin{array}{l}\mathbf{1 0}^{-\mathbf{4}} \\
\text { Iter. }\end{array}$ & $\begin{array}{c}\mathbf{1 0}^{-\mathbf{5}} \\
\text { Iter. }\end{array}$ & $\begin{array}{c}\mathbf{1 0}^{-\mathbf{2}} \\
\text { Time }\end{array}$ & $\begin{array}{c}\mathbf{1 0}^{-\mathbf{3}} \\
\text { Time }\end{array}$ & $\begin{array}{c}\mathbf{1 0}^{-\mathbf{4}} \\
\text { Time }\end{array}$ & $\begin{array}{c}\mathbf{1 0}^{-\mathbf{5}} \\
\text { Time }\end{array}$ \\
\hline$[0,0]^{T}$ & 16 & 220 & 2231 & 29253 & 0.21543 & 2.35401 & 29.86562 & 224.95083 \\
{$[10,10]^{T}$} & 27 & 190 & 2072 & 25762 & 0.25322 & 2.64742 & 26.84528 & 198.26446 \\
{$[-5,-5]^{T}$} & 43 & 411 & 3801 & 47891 & 0.78262 & 4.77116 & 42.41738 & 427.904781 \\
\hline
\end{tabular}

\section{Conclusions}

We have developed an extragradient-like method to solve pseudomonotone variational inequalities in real Hilbert space. The method had an explicit formula for an appropriate and effective stepsize evaluation on each step. For each iteration, the stepsize formula is modified based on the previous iterations. The numerical investigation was presented to explain the numerical effectiveness of our algorithm relative to other methods. These numerical studies suggest that viscosity schemes in this sense generally improve the effectiveness of the iterative sequence.

Author Contributions: Data curation, N.W.; formal analysis, T.K.; funding acquisition, N.P. (Nuttapol Pakkaranang), N.P. (Nattawut Pholasa) and T.K.; investigation, N.W., N.P. (Nuttapol Pakkaranang) and T.K.; methodology, T.K.; project administration, H.u.R., N.P. (Nattawut Pholasa) and T.K.; resources, N.P. (Nattawut Pholasa) and T.K.; software, H.u.R.; supervision, H.u.R. and N.P. (Nattawut Pholasa); Writing-original draft, N.W. and H.u.R.; Writing—review and editing, N.P. (Nuttapol Pakkaranang). All authors have read and agreed to the published version of the manuscript.

Funding: This research was funded by University of Phayao and Phetchabun Rajabhat University.

Acknowledgments: We are very grateful to the editor and the anonymous referees for their valuable and useful comments, which helps in improving the quality of this work. N. Wairojjana would like to thank by Valaya 
Alongkorn Rajabhat University under the Royal Patronage (VRU). N. Pholasa would like to thank by University of Phayao. T. Khanpanuk would like to thanks Phetchabun Rajabhat University.

Conflicts of Interest: The authors declare no conflict of interest.

\section{References}

1. Stampacchia, G. Formes bilinéaires coercitives sur les ensembles convexes. Comptes Rendus Hebd. Seances Acad. Sci. 1964, 258, 4413.

2. Konnov, I.V. On systems of variational inequalities. Russ. Math. C/C Izv. Vyss. Uchebnye Zaved. Mat. 1997, $41,77-86$.

3. Kassay, G.; Kolumbán, J.; Páles, Z. On Nash stationary points. Publ. Math. 1999, 54, 267-279.

4. Kassay, G.; Kolumbán, J.; Páles, Z. Factorization of Minty and Stampacchia variational inequality systems. Eur. J. Oper. Res. 2002, 143, 377-389. [CrossRef]

5. Kinderlehrer, D.; Stampacchia, G. An Introduction to Variational Inequalities and Their Applications; Society for Industrial and Applied Mathematics: Philadelphia, PA, USA, 2000. [CrossRef]

6. Konnov, I. Equilibrium Models and Variational Inequalities; Elsevier: Amsterdam, Netherlands, 2007; Volume 210.

7. Elliott, C.M. Variational and Quasivariational Inequalities Applications to Free-Boundary ProbLems. (Claudio Baiocchi And António Capelo). SIAM Rev. 1987, 29, 314-315. [CrossRef]

8. Nagurney, A.; Economics, E.N. A Variational Inequality Approach; Springer: Dordrecht, The Netherlands, 1999.

9. Takahashi, W. Introduction to Nonlinear and Convex Analysis; Yokohama Publishers: Yokohama, Japan, 2009.

10. Korpelevich, G. The extragradient method for finding saddle points and other problems. Matecon 1976, 12, 747-756.

11. Noor, M.A. Some iterative methods for nonconvex variational inequalities. Comput. Math. Model. 2010, 21, 97-108. [CrossRef]

12. Censor, Y.; Gibali, A.; Reich, S. The subgradient extragradient method for solving variational inequalities in Hilbert space. J. Optim. Theory Appl. 2010, 148, 318-335. [CrossRef]

13. Censor, Y.; Gibali, A.; Reich, S. Extensions of Korpelevich's extragradient method for the variational inequality problem in Euclidean space. Optimization 2012, 61, 1119-1132. [CrossRef]

14. Malitsky, Y.V.; Semenov, V.V. An Extragradient Algorithm for Monotone Variational Inequalities. Cybern. Syst. Anal. 2014, 50, 271-277. [CrossRef]

15. Tseng, P. A Modified Forward-Backward Splitting Method for Maximal Monotone Mappings. SIAM J. Control Optim. 2000, 38, 431-446. [CrossRef]

16. Moudafi, A. Viscosity Approximation Methods for Fixed-Points Problems. J. Math. Anal. Appl. 2000, 241, 46-55. [CrossRef]

17. Zhang, L.; Fang, C.; Chen, S. An inertial subgradient-type method for solving single-valued variational inequalities and fixed point problems. Numer. Algorithms 2018, 79, 941-956. [CrossRef]

18. Iusem, A.N.; Svaiter, B.F. A variant of korpelevich's method for variational inequalities with a new search strategy. Optimization 1997, 42, 309-321. doi:10.1080/02331939708844365. [CrossRef]

19. Thong, D.V.; Hieu, D.V. Modified subgradient extragradient method for variational inequality problems. Numer. Algorithms 2017, 79, 597-610. [CrossRef]

20. Thong, D.V.; Hieu, D.V. Weak and strong convergence theorems for variational inequality problems. Numer. Algorithms 2017, 78, 1045-1060. [CrossRef]

21. Censor, Y.; Gibali, A.; Reich, S. Strong convergence of subgradient extragradient methods for the variational inequality problem in Hilbert space. Optim. Methods Softw. 2011, 26, 827-845. [CrossRef]

22. Gibali, A.; Reich, S.; Zalas, R. Outer approximation methods for solving variational inequalities in Hilbert space. Optimization 2017, 66, 417-437. [CrossRef]

23. Ogbuisi, F.U.; Shehu, Y. A projected subgradient-proximal method for split equality equilibrium problems of pseudomonotone bifunctions in Banach spaces. J. Nonlinear Var. Anal. 2019, 3, 205-224.

24. Ceng, L.C. Asymptotic inertial subgradient extragradient approach for pseudomonotone variational inequalities with fixed point constraints of asymptotically nonexpansive mappings. Commun. Optim. Theory 2020, 2020, 2. 
25. Wang, L.; Yu, L.; Li, T. Parallel extragradient algorithms for a family of pseudomonotone equilibrium problems and fixed point problems of nonself-nonexpansive mappings in Hilbert space. J. Nonlinear Funct. Anal. 2020, 2020, 13.

26. Ceng, L.C. Two inertial linesearch extragradient algorithms for the bilevel split pseudomonotone variational inequality with constraints. J. Appl. Numer. Optim. 2020, 2, 213-233. [CrossRef]

27. Ur Rehman, H.; Kumam, P.; Je Cho, Y.; Suleiman, Y.I.; Kumam, W. Modified Popov's explicit iterative algorithms for solving pseudomonotone equilibrium problems. Optim. Methods Softw. 2020, 1-32. [CrossRef]

28. Ur Rehman, H.; Kumam, P.; Kumam, W.; Shutaywi, M.; Jirakitpuwapat, W. The inertial sub-gradient extra-gradient method for a class of pseudo-monotone equilibrium problems. Symmetry 2020, 12, 463. [CrossRef]

29. Ur Rehman, H.; Kumam, P.; Argyros, I.K.; Deebani, W.; Kumam, W. Inertial extra-gradient method for solving a family of strongly pseudomonotone equilibrium problems in real Hilbert spaces with application in variational inequality problem. Symmetry 2020, 12, 503. [CrossRef]

30. Ur Rehman, H.; Kumam, P.; Argyros, I.K.; Alreshidi, N.A.; Kumam, W.; Jirakitpuwapat, W. A self-adaptive extra-gradient methods for a family of pseudomonotone equilibrium programming with application in different classes of variational inequality problems. Symmetry 2020, 12, 523. [CrossRef]

31. Ur Rehman, H.; Kumam, P.; Shutaywi, M.; Alreshidi, N.A.; Kumam, W. Inertial optimization based two-step methods for solving equilibrium problems with applications in variational inequality problems and growth control equilibrium models. Energies 2020, 13, 3292. [CrossRef]

32. Ur Rehman, H.; Kumam, P.; Abubakar, A.B.; Cho, Y.J. The extragradient algorithm with inertial effects extended to equilibrium problems. Comput. Appl. Math. 2020, 39, 100. [CrossRef]

33. Antipin, A.S. On a method for convex programs using a symmetrical modification of the Lagrange function. Ekonomika Matematicheskie Metody 1976, 12, 1164-1173.

34. Migórski, S.; Fang, C.; Zeng, S. A new modified subgradient extragradient method for solving variational inequalities. Appl. Anal. 2019, 1-10. [CrossRef]

35. Yang, J.; Liu, H.; Liu, Z. Modified subgradient extragradient algorithms for solving monotone variational inequalities. Optimization 2018, 67, 2247-2258. [CrossRef]

36. Kraikaew, R.; Saejung, S. Strong Convergence of the Halpern Subgradient Extragradient Method for Solving Variational Inequalities in Hilbert Spaces. J. Optim. Theory Appl. 2013, 163, 399-412. [CrossRef]

37. Heinz H. Bauschke, P.L.C. Convex Analysis and Monotone Operator Theory in Hilbert Spaces, 2nd ed.; CMS Books in Mathematics; Springer International Publishing: Cham, Switzerland, 2017.

38. Goebel, K.; Reich, S. Uniform Convexity, Hyperbolic Geometry, and Nonexpansive Mappings; Marcel Dekker, Inc.: New York City, NY, USA, 1984.

39. Cottle, R.W.; Yao, J.C. Pseudo-monotone complementarity problems in Hilbert space. J. Optim. Theory Appl. 1992, 75, 281-295. [CrossRef]

40. Maingé, P.E. Strong Convergence of Projected Subgradient Methods for Nonsmooth and Nonstrictly Convex Minimization. Set-Valued Anal. 2008, 16, 899-912. [CrossRef]

41. Takahashi, W. Nonlinear Functional Analysis; Yokohama Publishers: Yokohama, Japan, 2000.

42. Liu, Z.; Zeng, S.; Motreanu, D. Evolutionary problems driven by variational inequalities. J. Differ. Equ. 2016, 260, 6787-6799. [CrossRef]

43. Liu, Z.; Migórski, S.; Zeng, S. Partial differential variational inequalities involving nonlocal boundary conditions in Banach spaces. J. Differ. Equ. 2017, 263, 3989-4006. [CrossRef]

44. Hu, X.; Wang, J. Solving pseudomonotone variational inequalities and pseudoconvex optimization problems using the projection neural network. IEEE Trans. Neural Networks 2006, 17, 1487-1499. [CrossRef]

45. Shehu, Y.; Dong, Q.L.; Jiang, D. Single projection method for pseudo-monotone variational inequality in Hilbert spaces. Optimization 2018, 68, 385-409. [CrossRef]

(C) 2020 by the authors. Licensee MDPI, Basel, Switzerland. This article is an open access article distributed under the terms and conditions of the Creative Commons Attribution (CC BY) license (http:/ / creativecommons.org/licenses/by/4.0/). 